\title{
Seguimiento de pacientes con hiperfenilalaninemia diagnosticados precozmente
}

\author{
Verónica Cornejo E. ${ }^{1}$; Erna Raimann B. ${ }^{2}$; \\ Ximena Godoy P. ${ }^{3}$; Marta Colombo $\mathrm{C}^{2}$
}

Early diagnosis and management of hyperphenylalaninemia

\begin{abstract}
Seventeen newborn oalients with serum phenylalanine concentrations 16.5 to $46.2 \mathrm{mg} / \mathrm{dl}$ are described to enhance local interest in phenylketonuria screening and early mancgement. Diagnosis was comlirmed at age $20.3 \pm$ 12.0 days and before age 30 days with only hwo exeptions. All patients storled dielary treatment at cliagnosis and have beer sollowed up from then on at an University of Chi.e's Instifute for Nutrilion and Food Tuchnology oukpatiert clinis Serum phenylalanine concentrations along follow up have been crround 2 to $6 \mathrm{mg} / \mathrm{dl}$ even though tronsierit noncompliances with diekary restrictions were associated with serum levels of $18 \mathrm{mg} / \mathrm{dl}$. Psychomotor development has been rormal to date in fifeen oatienis, one potient has some slighl relardation which is at leost partially reloted to low social and cultural conditicns, while the other one is under study because of a hypotonic syndrame. Body growth nas teen normal exept by one patient which required ealy heart surgery for lrcuspid atresia and the one afected by the hypotonic syndrome.
\end{abstract}

(Key words: biopterin, dihydropteridine reduclase, phenylalanine, penylalanirie hydroxylese, phenylketonuria.)

La mayoría de las hiperfenilalaninemias son debidas a alteraciones en el metabolismo del aminoácido esencial fenilalanina a tirosina, por deficiencia o ausencia de la enzima fenilalanina hidroxilasa (FAH). En 1 a $2 \%$ de los casos el defecto se origina en déficit de cofactores (pterinas) de esta enzima, por escasez de dihidropteridina reductasa. La clasificación de las hiperfenilalaninemias se basa en las manifestaciones clínicas, la actividad enzimática residual y la mutación existente. La deficiencia enzimática causa aumento de la concentración de fenilalanina en los jíquidos corporales y el empleo de vías alternas que conducen a síntesis de fenilquetonas (ácidos fenilpirúvico, fenil-acéti-

1. Nutricionista, Magister en Nutrición Humana. Unidad de Genética y Enfermedades Metabólicas, Instituto de Nutrición y Tecnología de los Alimentos (LNTA), Untiversidad de Chile.

2. Unidad de Genética y Enferrnedades Metabólicas, Ins. tituto de Nutrición y Tecnología de los Alimentos (INTA). Universidad de Chile.

3. Psicóloga Unidad de Genélica y Enfermedades Metabólicas, Insticuto de Nutrición y Tecnologia de los Aliinentos (INTA), Universidad de Chile. co y fenil-láctico). Todos los tipos de hiperfenilalaninemia se heredan de modo autosómico recesivo, ambos padres son portadores $y$ hay $25 \%$ de riesgo de recurrencia en los hermanos ${ }^{2}$. $\mathrm{Su}$ incidencía es muy variable, entre 1:10000 recién nacidos caucásicos y 1:120000 japone$\operatorname{ses}^{3,4}$.

La forma más severa es la fenilquetonuria clásica, descrita en 1934 por Fölling, que se caracteriza por actividad de $\mathrm{FAH}$ menor a $1 \%$; concentraciones de fenilalanina en sangre y tejidos de más de $20 \mathrm{mg} / \mathrm{dl}$, tirosina sérica mayor a $5 \mathrm{mg} / \mathrm{dl}$ y exceso de fenilquetonas en la orina. En la hiperfenilalaninemia persistente, la concentración sérica de fenilalanina es de 4 a 19 $\mathrm{mg} / \mathrm{dl}$, puede no haber fesilquetonuria, la tirosinemia es normal y la actividad enzimática de FAH es 3 a $50 \%$ de 10 esperado. En la forma benigna, la actividad enzimática es mayor a $50 \%$, la fenilalanina sérica, que está entre 4 y 10 $\mathrm{mg} / \mathrm{dl}$, puede normalizarse a los seis meses de edad; la tirosinemia es normal. En la deficiencia de biopterina, la fenilalaninemia es mayor a $4 \mathrm{mg} / \mathrm{dl}$, la relación biopterina/neopterina es anormal, no hay respuesta al tratamiento 
nutricional y el daño neurológico ocurre precozmente.

La fenilquetonuria clásica no tratada causa deterioro neurológico progresivo y retardo mental profundo. En $25 \%$ de los afectados ocurren convulsiones del tipo espasmos masivos, $78 \%$ a 95\% tienen alteraciones en el electroencefalograma (EEG) y trastornos psiquiátricos que incluyen conductas psicóticas y automutilación. Una de sus características clínicas es la despigmentación de la piel y fanéreos, debida a la alteración del metabolismo de la tirosina, eczema rebelde al tratamiento y olor a humedad causado por acumulación de fenilquetonas 5 .

A partir de 1963 , con los programas de detección neonatal, tratamiento nutricional pre$\operatorname{coz}$ y seguimiento estricto, la fenilquetonuria ha dejado de ser causa de retardo mental en los países de mayor desarrollo ${ }^{6-8}$. En Chile el rastreo neonatal de fenilquetonuria se empezó a aplicar en 1988 en $18 \%$ de los recién nacidos de Santiago (la ciudad capital) ${ }^{9}$, posteriormente se amplió a toda la Región Metropolitana de Santiago con coberturas de $98 \%$ y, dentro de poco, se pretende cubrir a todos los 310000 nacidos cada año en el país ${ }^{\mathbf{1}}$. La experiencia ha permitido detectar, hasta la fecha, 17 recién nacidos con hiperfenilalaninemia, los que han sido seguidos en el policlínico de enfermedades metabólicas del Instituto de Nutrición y Tecnologia de los Alimentos (INTA), de la Universidad de Chile, cuya evolución se describe en seguida.

\section{Material y Método}

Diecisiete niños con hiperfenilalaninemia fueron detectados entre los atos 1988 y 1993 , en el período neona. tal, mediante el método de inhibición bacteriana o prueba de Guthrie (con corte de normalidad $<2 \mathrm{mg} / \mathrm{dl}$ de fenilalaпiлa) ${ }^{11}$, doce en el programa masivo de rastreo de la Región Metropolitana y otros cinco en exámenes de rutina realizados por clínicas privadas. El diagnóstico se confirm6 por cromatografía líquida de alta presión ${ }^{12}$. Las determinaciones posceriores de fenilalaninemia. durante el seguimiento, se realizaron también por inhibición bacteriana.

Una vez confirmado el diagnóstico, los pacientes ingresaron a un programa de segnimiento integral, con dieta, actividades de educación, evaluaciones clínicas, nutricionales. bioquímicas, neurológicas y psicológicas frecuentes. La educacion nutrícional fue reforzada con folletos educativos sobre la enfermedad, su tralamiento y el contenido de fenilalanina de los alimentos. La dieta, restringida en fenilatanina, se describe en la tabla, e inciuyó los suplementos necesarios de vitaminas, oligoelementos y minera-
Jes, de acuerdo a las Recomendacjones para Raciones Dietéticas en paciences con enfermedades metabólicas (RDA) ${ }^{13}$. Perídicamente se realizaron cálculos autricionales mediante un programa para ordenadores (Aminoacid Analyzer, versión 2.4, IBM, EUA) para modificar las indicaciones correspondientes. Se realizaron evaluaciones psicométricas mediante prueba de Bayley para los niños menores de 30 meses $^{14}$ y Stanford-Binet en los mayores ${ }^{16}$. El estado nutricional se determinó de acuerdo a las tablas de peso para la edad y talla para la edad del Cenıro Estadounidense de Estadísticas de Salud (NCHS).

\section{Resultados}

El diagnóstico de hiperfenilalaninemia se estableci 6 a los $20,29 \pm 12,6$ días de edad (mátgenes 10 a 60 días) en casi todos los casos. Sólo en dos se hizo después de los 30 días: uno a los 60 , porque la obtención de la muestra se atras 6 debido a que fue sometido a cirugía cardiovascular (sufría de atresia tricuspídea y comunicación interventricular) y otro a los 40 días, proveniente de una clínica privada, lo que retardó la prueba de confirmacion. En todos la concentración sérica de fenilalanina era mayor a 2 $\mathrm{mg} / \mathrm{dl}$ en el primer examen y se confirmó el diagnóstico, obteniéndose concentraciones de $27,14 \pm 8,65 \mathrm{mg} \%$ (mediana 26,0; márgenes 18,0 a $46,2 \mathrm{mg} \%$ ) por cromatografía. Como consecuencia de la identificación precoz de dos de estos pacientes, se detectaron otros dos casos, de 15 meses y 5 años de edad, en sendos hermanos. Otro paciente tiene antecedente de

\section{Tabla}

Recomendación de nutrientes para niños con fenilquetonuria

$$
\text { Nutrientes }
$$

\begin{tabular}{cccc}
$\begin{array}{c}\text { Edad } \\
\text { (meses) }\end{array}$ & $\begin{array}{c}\text { Fenilalanina } \\
\text { (mg/kg/día) }\end{array}$ & $\begin{array}{c}\text { Proteínas } \\
\text { (g/kg/día) }\end{array}$ & $\begin{array}{c}\text { Calorías } \\
\text { (cal/kg/día) }\end{array}$ \\
\hline $0<3$ & 55 & $3,0-2,50$ & 120 \\
$3<6$ & 35 & $3,0-2,50$ & 115 \\
$6<9$ & 30 & $2,5-2.25$ & 110 \\
$9<12$ & 25 & $2,5-2.25$ & 105 \\
\hline Edad & & & \\
(años) & (mg/día) & (g/día) & (cal/días) \\
\hline $1<4$ & 325 & 25 & 1300 \\
$4<7$ & 425 & 35 & 1700 \\
$7<11$ & 450 & 40 & 2400 \\
\hline
\end{tabular}

Fuente: RDA 1989 
una familiar con fenilquetonuria. La edad de los pacientes a la fecha de este análisis era 18,8 \pm 12,7 meses (márgenes 6 a 48 meses).

La dieta que recibieron los afectados aportó $105,4 \pm 18,0 \mathrm{cal}, 1,6 \pm 0,45 \mathrm{~g}$ proteína y 28,9 $\mathrm{mg} \pm 14,8$ fenilalanina por $\mathrm{kg}$ - día (tabla). Los aportes de otros nutrientes fueron: vitamina B12 $(74 \%)$, calcio $84,8 \%$, fierro $71,43 \%$, zinc $47,86 \%$, magnesio $95,28 \%$ y fosforo $70,75 \%$ de las recomendaciones dietéticas citadas. Las concentraciones séricas de fenilalanina fluctuaron durante el seguimiento entre 2 y $18 \mathrm{mg} / \mathrm{dl}$ (media $7,5 \mathrm{mg} / \mathrm{dl}$ ) , pero en la mayoría de los pacientes se han mantenido en márgenes aceptados para casos de hiperfenilalaninemia (2 a 6 $\mathrm{mg} / \mathrm{dl}$ ), si bien en dos niños que cometieron transgtesiones por corto tiempo llegaron a 18 $\mathrm{mg} / \mathrm{dl}$ de suero.

El crecimiento en peso y talla ha cursado dentro de los márgenes normales en 15 pacientes $(82,2 \%)$. Uno de los dos casos en que ha sido insuficiente es en el niño con malformaciones congénitas del corazón, quien se encuentra en los percentilos 5 y 10 de peso y talla para edad, respectivamente; el otro está siendo estudiado, ya que presentó, en el segundo semestre de su vida, desaceleración del crecimiento y síndrome hipotónico, manteniéndose su peso y talla en el percentil 5 para la edad.

En la evaluación psicométrica 15 niños $(82,2 \%)$ obtuvieron resultados considerados normales, con coeficientes de desarrollo mental de $92,3 \pm 10,2$ (mediana 91 , márgenes 71 a 114) $y$ de desarrollo motor de $89,9 \pm 15,0$ (mediana 91, márgenes 58 a 113). Sólo dos de los niños sometidos a las pruebas de Bayley o las de Stanford Binet, muestran déficit psicomotor global, uno proviene de un área rural de exirema pobreza, el otro es el niño en estudio por síndrome hipotónico. Tres lactantes mostraron cuocientes de desarrollo motor por debajo del promedio, sin embargo el desarrollo mental de estos niños es normal.

\section{Comentario}

En el Centro de Referencia para Diagnóstico y Tratamiento de Enfermedades Metabólicas en el INTA, Universidad de Chile, se ha confirmado el diagnóstico de hiperfenilalaninemia en 146 pacientes, desde su creación en $1973^{15}$. El promedio de edad de diagnóstico en esta experiencia es comparable con el de otros países ${ }^{1 ?}$. Los casos detectados después de los 30 días refuerzan la recomendación de tomar muestras en tarjeta de papel filtro en los plazos convencionales, como también, y con mayor razón, en recién nacidos enfermos ${ }^{18}$. Debido a la herencia autosómica recesiva de la hiperfenilalaninemia, la educación y el consejo genético de toda la familia son imprescindibles, como se ilustra con la identificación tardía de dos hermanos mayores afectados a raíz de la detección neonatal de pacientes de la enfermedad.

Con el tratamiento dietético de la fenilquetonuria se logra reventir los síntomas y las alteraciones bioquímicas de la afección. En la práctica, el tratamiento nutricional comienza cuando el niño tiene concentraciones séricas de fenilalanina de $10 \mathrm{mg} / \mathrm{dl}$ o más con una dieta normal, ya que es sobre esta tasa que se produce deterioro del sistema nervioso centrall. 2 recomendándose que la dieta contenga 200 a 500 $\mathrm{mg}$ - día del aminoácido, ajustando su ingestión para mantener concentraciones plasmáticas entre 2 y $6 \mathrm{mg} / \mathrm{dl}$. La tolerancia a la fenilalanina parece depender del tipo de hiperfenilalaninemia del paciente ${ }^{13}$.

La baja ingestión de fenilalanina requerida por los niños de esta serie sugiere que la mayoría de ellos pudiese corresponder a casos de ferilquetonuria clásica. De tres niños que están con ingestas de fenilalaninemia mayores a 35 $\mathrm{mg}$ - kg • día, dos tenían concentraciones séricas iniciales del aminoácido 18 y $19,4 \mathrm{mg} / \mathrm{dl}$ respectivamente, por to que pudiesen ser casos de hiperfenilalaninemia persistente. El tercero es un lactante menor, razón por la cual sus requerimientos son mayores. La dieta recomendada para estos pacientes no es capaz, por si sola, de cubrir las recomendaciones de vitaminas y minerales, especialmente $\mathrm{Fe}, \mathrm{Ca}, \mathrm{Zn}, \mathrm{Mg}, \mathrm{P}$, vitamina $\mathrm{B} 12$. Por esta razón se recomiendan suplementos de ellos ${ }^{19}$. En nuestra serie el mineral más deficitario con respecto a las recomendaciones es el zinc.

Las alteraciones detectadas en el desarrollo mental no son forzosamente atribuíbles a 1 a enfermedad metabólica en esta serie. El diagnóstico precoz y un programa integral de seguimiento permiten prevenir el retardo mental y promover el desarrollo normal en los pacientes con hiperfenilalaninemia. 


\section{Resumen}

Se presentan 17 pacientes con hiperfenilalaninemia diagnosticados en el período neonatal $(20,3 \pm 12,6$ días de vida), cuyas concentraciones iniciales de fenilalanina sérica eran de 16,5 a $46,2 \mathrm{mg} \%$. En todos el tratamjento dietético comenzó al hacer el diagnóstico y han sido seguidos en un policlínico especial del Instituto de Nutrición y Tecnología de los Alimentos de la Universidad de Chile. Se describen la dieta, los suplementos de minerales y vitaminas requeridos, que han permitido, durante el seguimiento, mantener concentraciones de fenilalanina en el suero entre 2 y $6 \mathrm{mg} / \mathrm{d}$ ), salvo transgresiones transitorias que han ocurrido en dos casos. En $82,2 \%$ de los casos el crecimiento y el desarrollo han sido normales. Se subraya la importancia de tomar la primera muestra en tarjeta de papel filtro a todos los recién nacidos antes de cumplir 10 días de vida, para hacer oportunamente el diagnóstico. Los programas de seguimiento integral facilitan el manejo y el desarrollo físico y mental normales de estos niños.

(Palabras clave: biopterina, dihidropteridina reductasa, fenilalanina, fenilalanina hidroxilasa, fenilquetonuria.)

\section{Referencias}

I. Scriver Ch, Kutman $S$ : The hyperphenyladaninemias. Chapter 15. In: Scriver Ch, Beaudet A, Sly W, Valle D (ed). The metabolic basis of inherited diseases. Tome 1. 6th edition. McGraw-Hill Information Services Company 1989: 495-546.

2. Rosemberg $/ E, S c r i v e r ~ C R$ : Disorders of amino acids metabolism. In: Bondy PK, Rosemberg GW. (ed). Duncan's diseases of metabolism. W.B. Saunders, Philadelphia, London and Toronto 1974: 497-610.

3. Holtsman $N$, Meck A, Mellts $S$ : Neonatal screening for phenylketonuria. I. Effectiveness JAMA. 1974; 229: $667-670$.

4. Horst $\mathrm{J}$ : Impact of systematic screening for PKU. In: Farriaux JP, Dhondt JL (ed), New Horizons in neonatal screening. Elsevier Science B.V. Excerpta Medica. Amsterdam. London, New York. Tokyo. 1994: 101-105.

5. Menkes, JH: The pathognesis of mental retardation in phenylketonuria and other inbom errors of amino acid metabolism. Pediatrics 1967; 39: 297-302.

6. Gutter $F$, Lou $H$ : Phenylketonuria and hyperphenylalaninemia. In: Fernández J, Saudubray IM, Tada K. (eds). fribon metatolic diseases. SpringerVerlag. Berlin, Heidelberg. New York 1990: I61173

7. Smith I, Beasley MG. Ades AE: Intelligence and quality of dietary in phenylkelonuria. Arch Dis Child 1990; 65: 472-478.

8. Michals $X$, Azen C, Acosta $P$, Koch $R$, Matalon $R$ Blood phenylalanin levels and intelligence of 10 -yearold childred with PKU in the nalional collaborative study. J Am Diet Assoc 1988: 88; 1226-1229.

9. Carnejo V. Raimann E, Moraga M: Programa de ratreo neonatal de fenilquetonuria. Rev Chil Pediatr 10090; 61: 309-312.

10. Repubilica de Chile: Ministerio de Economí, Fomento y Reconstrucción. Instituto Nacional de Estadísticas, Demografía. Santiago, Chile 1992.

11. Guthrie R: Phenylketonurja screening programs. New Engl J Med 1983; 269: 52-58.

12. HiII D, Bunsworth L, Skea W. Pfeifer R: Quantitative HPLC analyzes of plasma aminoacids as orthothaldialdehyde ethanethiol derivate. J Liquid Chromathography 1982; 5: 2369-2393.

13. Francis $D$ : Dietary management of hyperphenylalaninemia. In: Clayton $F$ (ed). Diets for Sick Children, chapter 19. Blackwell Scientific Publications, 4rth ed. Loudon. 1987.

14. Bayley N: Bayley scale of infant development. Birth to 42 months. San Antonio the Psycological Corporation Harcout Brace and Company, USA, 1993.

15. Cotombo $M$. Raimann E. Cornejo V: Ertores congénitos del metabolismo. Cap. UJ. En: Winter A, Puentes $R$ (eds). Del recién nacido al adolescente. Medicina infantil. Tomo I. Speed Printer Santiago, Chile. 1991: 206-235.

16. Terman L. Merrill M: Medida de la inteligencia. 9th Espasa-Calpe S.A. Madrid, España. 1975.

17. Frecheste A, Russo $P$ : Prospective study of early neonatal screening for phenylketonuria. New Engl J Med 1981: 304: 294-296.

18. $M c$ Cabe RB: Screening for PKU in sick or premature neonates. J Pediatr 1983; 103: 502-503.

19. Acosta P: Nutrition studies in treated infants with phenylketonuria. Int Pediatr 1993; 8: 63-73. 